\title{
Proposta de uma abordagem psicoeducacional em grupos para pacientes adultos com Transtorno de Déficit de Atenção/Hiperatividade
}

\author{
Eugenio Horácio Grevet* \\ Paulo Belmonte de Abreu** \\ Flávio Shansis***
}

\section{INTRODUÇÃO}

O Transtorno de Déficit de Atenção/Hiperatividade (TDAH) é um transtorno psiquiátrico que se caracteriza pela presença de desatenção, hiperatividade e impulsividade (American Psychiatric Association, 1994). O DSM-IV ${ }^{1}$ exige a presença de, pelo menos, seis sintomas de uma lista de nove de desatenção e/ou seis sintomas de uma lista de nove de hiperatividade-impulsividade para que o diagnóstico seja estabelecido em crianças, adolescentes ou adultos. A partir destes critérios, são identificadas três tipos de apresentações: TDAH subtipo desatento, subtipo hiperativo e subtipo combinado (desatento e hiperativo).

\footnotetext{
* Pesquisador do Ambulatório de Déficit de Atenção/Hiperatividade no Adulto do Hospital de Clínicas de Porto Alegre / Universidade Federal do Rio Grande do Sul (HCPA/UFRGS), Mestre em Bioquímica pela UFRGS, Doutorando da Pós-Graduação em Psiquiatria da FAMED-UFRGS.

** Professor Adjunto do Departamento de Psiquiatria e Medicina Legal da FAMED-UFRGS e Coordenador do Ambulatório de Déficit de Atenção/ Hiperatividade no Adulto do HCPA/UFRGS.

${ }^{* * *}$ Médico Contratado do Serviço de Psiquiatria do Hospital de Clínicas de Porto Alegre, Mestre em Bioquímica pela UFRGS, Doutorando da pósGraduação em Bioquímica da UFRGS.
}

\begin{abstract}
A prevalência deste transtorno na população infantil é estimada em 4,5 a $9,0 \%$ para o subtipo desatento, 1,7 a $3,9 \%$ para o subtipo hiperativo e impulsivo, e de 1,9 a $4,8 \%$ para 0 subtipo combinado. A persistência deste quadro ao longo da vida adulta ocorre em torno de $50 \%$ daqueles indivíduos que preenchiam os critérios para o TDAH na sua infância ${ }^{2}$. Entretanto, chama a atenção que até $90 \%$ das pessoas que apresentavam este transtorno na infância podem permanecer com sintomas isolados de desatenção sem preencher na totalidade os critérios formalmente necessários pelo DSM-IV para este transtorno na vida adul$\mathrm{ta}^{3}$.

O TDAH causa grande impacto na vida de seus portadores. Estes apresentam relações inter-pessoais instáveis e tumultuadas, baixo desempenho acadêmico e profissional, o que acaba por acarretar enormes prejuízos no funcionamento familiar e social. Este impacto é ainda amplificado pelas altas taxas de comorbidades com outras doenças psiquiátricas, pois sabe-se que $77 \%$ dos adultos com TDAH apresentam outro transtorno psiquiátrico concomitante. Os transtornos comórbidos mais comuns
\end{abstract}


são: Depressão Maior, Transtornos de Ansiedade, Transtornos de Humor Bipolar, Transtornos de Abuso de Substâncias, Transtornos de Personalidade e alterações de conduta na idade adulta 4 .

A etiologia do TDAH tem sido objeto de investigação nas áreas da neuroquímica, neuranatomia e genética. A resposta ao metilfenidato - que age nos sistemas noradrenérgico ou dopaminérgico - sugere uma possível alteração nos sistemas destes neurotransmissores . Alterações em circuitos neuronais na área fronto-estriatal parecem estar envolvidas na etiologia e na manutenção deste transtorno. Ainda, estudos com gêmeos monozigóticos evidenciaram uma concordância de $70 \%$, sugerindo fortemente a existência de bases genéticas.

Os pacientes portadores de TDAH apresentam alterações especificas em uma função cognitiva chamada de Função Executiva (FE). Esta é a função mental que coordena a memória imediata, memória imediata verbal, autoregulação dos afetos e permite a reconstituição e análise do próprio comportamento. Alterações nesta função podem acarretar um menor controle dos impulsos, dificuldades de reter informações, respostas verbais inadequadas e problemas no controle motor a estímulos. Alguns autores consideram a FE o "maestro da sinfonia mental do indivíduo"5. Todas as pessoas - com ou sem TDAH - podem apresentar algum grau de alteração da FE. Entretanto, o TDAH pode ser entendido como o extremo de um comportamento e de um mau funcionamento da FE, uma vez que esta população apresenta um grande número de problemas na área dos processos de ensino/aprendizado ${ }^{6}$, o que acarreta dificuldades na aquisição de novos conteúdos.

A partir desta compreensão do TDAH como uma disfunção executiva - com conseqüentes dificuldades na aquisição de novos conhecimentos -, o objetivo do presente artigo, é o de propor uma nova abordagem psicoeducativa que leve em consideração técnicas pedagógicas na criação de grupos psicoeducativos para pacientes adultos portadores de TDAH.

\section{TRATAMENTO}

O tratamento desse transtorno exige uma abordagem ampla do paciente. A avaliação deve incluir aspectos físicos, emocionais e sociais uma vez que o TDAH apresenta inúmeros problemas associados em diferentes áreas da vida do paciente. $O$ tratamento para o TDAH inclui as abordagens farmacológica e psicote- rápica. O tratamento farmacológico clássico (que não será abordado neste artigo) baseia-se na utilização de psicoestimulantes para os pacientes com TDAH, sejam eles crianças, adolescentes ou adultos. Não é mais admissível, nos dias atuais, que um paciente com TDAH não receba medicação, uma vez que se sabe que a dimensão da desatenção responde muito pouco, ou quase nada, às abordagens psicoterápicas. Contudo, os tratamentos psicoterápicos possuem um papel fundamental na recuperação do paciente ${ }^{7}$.

\subsection{Abordagens Psicossociais}

Os tratamentos psicoterápicos não são considerados de primeira linha para o TDAH. Entretanto, possuem importância fundamental no sentido de que podem informar ao paciente sobre sua condição, podendo, também ,melhorar seu desempenho cognitivo e comportamental nas situações e contextos onde os sintomas são mais prejudiciais.

As abordagens psicossociais que são utilizadas atualmente foram desenvolvidas para crianças e adolescentes. Sua utilização em adultos é uma adaptação destes métodos e, portanto, precisam ser ainda devidamente validadas.

Os tipos de psicoterapias que são utilizadas em crianças e adolescentes - baseadas em estudos clínicos controlados - são: a terapia cognitivo-comportamental individual, a terapia cognitivo-comportamental para familiares, a terapia de apoio para pacientes e familiares, o treinamento comportamental para familiares, 0 biofeedback, a biblioterapia e o treinamento de habilidades sociais para pacientes e familiares $^{8-14}$. Estes tratamentos mostraram-se bastante eficientes na redução da hiperatividade e dos problemas de conduta, porém o mesmo não ocorreu em relação aos sintomas de desatenção.

Em adultos, apenas um trabalho demonstrou que a Terapia Cognitivo-comportamental (TCC) pode ser útil na redução dos sintomas de TDAH, depressão e ansiedade. Os autores deste trabalho ${ }^{15}$ utilizaram a técnica de TCC, originalmente proposta para Depressão Maior por Aaron Beck, modificada para TDAH. O tratamento durou em média 11 semanas.

$\mathrm{Na}$ abordagem psicoterápica em crianças e adolescentes, o foco está voltado para o tratamento dos sintomas e para ajudar os familiares a lidarem com a criança. Por sua vez, o foco na psicoterapia do TDAH em adultos visa à aceitação da doença, uma vez que, em geral, os 
pacientes adultos com este transtorno sofreram durante anos as seqüelas do TDAH crônico. Os problemas mais comumente abordados em psicoterapias com estes pacientes são a autoestima, as relações conjugais, as dificuldades no trabalho e nas atividades acadêmicas. Geralmente, as abordagens psicossociais se iniciam por medidas psicoeducacionais.

\subsection{Abordagens Psicoeducacionais}

Este tipo de abordagem geralmente visa a ajudar o paciente a reconhecer os sintomas de sua doença, interpretar os danos causados pela mesma e planejar estratégias de convívio com a doença. Nesta etapa, os pacientes sentem-se reconfortados por terem, pela primeira vez, uma explicação para seus problemas. O paciente também pode se beneficiar tendo acesso a materiais impressos contendo informações detaIhadas sobre a doença, assim como hoje em dia pela internet ${ }^{11}$. Existem no mercado livros para o público leigo que oferecem uma boa gama de informações ${ }^{16,17}$, assim como muitos sites sobre o tema.

Um problema importante neste tipo de abordagem é a falta de guias técnicos que orientem a abordagem do ponto de vista pedagógico. Existem na literatura referências a técnicas psicoeducacionais que simplesmente apresentam aulas expositivas a respeito de conhecimentos técnicos da doença ${ }^{18}$. São raros os trabalhos que pormenorizam as técnicas pedagógicas empregadas, o tipo de aula, a duração, a freqüência, a avaliação da efetividade e a comparação com medidas controle.

Para outros transtornos, já há trabalhos como o de Anderson, Hogarty e Reiss ${ }^{19}$. Estes autores descrevem pormenorizadamente as técnicas psicoeducativas utilizadas no tratamento de famílias que apresentavam um membro com Transtorno do Humor ou Esquizofrenia. Eles propõem os seguintes objetivos para uma abordagem psicoeducativa:

- Capacitar o paciente e sua família a aceitar o fato de que o paciente sofre de uma doença médica que produz sintomas que afetam a auto-estima, o humor, a fala, o sono, o apetite e o comportamento social e sexual;

- Ensinar o paciente e sua família a identificar e caracterizar sintomas específicos que ocorrem no início de um episódio;

- Demonstrar para a família que os episódios da doença têm, inevitavelmente, um impacto sobre seu estilo de vida;

- Examinar as mudanças de papel que 448 mente o papel do paciente na família;

- Transmitir a idéia que as doenças do humor são "transtornos familiares" e auxiliar os familiares a distinguir casos da doença em outros familiares;

- Explicar as vantagens do tratamento preventivo a longo prazo;

- Explicar as vantagens do monitoramento laboratorial a longo prazo e encorajar a família a compartilhar as decisões terapêuticas com o paciente;

- Auxiliar a família a distinguir os efeitos colaterais das medicações e diferenciá-los dos sintomas da doença.

Os mesmos autores propõem que o atendimento seja feito em grupos e que sejam realizadas cinco sessões. A primeira sessão seria utilizada para discutir as crenças da família a respeito da doença. Esta sessão seria apenas para entender as teorias familiares sobre a doença, sem interferência da equipe. Na segunda sessão, são apresentadas as informações adequadas sobre a etiologia, curso, sintomas e tratamento. A segunda sessão tem um formato de perguntas e respostas abertas. Geralmente ocorrem acaloradas discussões nesta fase do tratamento, principalmente em relação às causas biológicas da doença. Em sessões subseqüentes, os pacientes são encorajados a relatar as suas vivências subjetivas dos sintomas. Na quinta sessão - a de encerramento -, é revisado o que a família aprendeu a respeito da doença e são discutidas estratégias para lidar com futuras crises.

De maneira geral, este tipo de abordagem poderia ser bem empregado em paciente com Transtorno de Déficit de Atenção/Hiperatividade com pequenas modificações que visem a ajustar a técnica às características da doença.

\section{PROPOSTA DE UMA ABORDAGEM PSICOEDUCAIONAL PARA O TDAH}

O tratamento psicoeducacional pode ser realizado individualmente ou em grupo. No tratamento individual, o paciente pode ser ajudado a lidar com a desatenção, a hiperatividade e a impulsividade, assim como a ter um controle melhor dos efeitos colaterais das medicações que está utilizando. O atendimento em grupo facilita o paciente a descobrir que ele faz parte de um grupo de indivíduos com problemas específicos e que, com estes, compartilha suas dificuldades, sintomas e problemas familiares. Em paises de poucos recursos - como o nosso - este último tipo de tratamento é o mais indica- 
do, já que otimiza a utilização de recursos financeiros e profissionais que são geralmente escassos.

A família pode entender melhor o comportamento dos seus membros afetados, pois muitas pessoas costumam fazer um julgamento moral dos sintomas de TDAH. É importante desfazer tais crenças distorcidas. Por exemplo, muitas esposas/esposos de pacientes com TDAH não suportam mais os sintomas do cônjuge. Os sintomas do paciente muitas vezes parecem indicar pouco interesse por parte do indivíduo afetado pelas pequenas coisas do dia-a-dia. Em muitos casos, os indivíduos afetados podem evitar conversas por achá-las desagradáveis. A falta de atenção com aspectos do dia-a-dia, como a educação dos filhos ou o esquecimento de pagar as contas no dia certo, podem ser o motivo de intermináveis discussões. Muitas vezes recomendações simples, que poucos casais seguem, podem aliviar 0 estresse conjugal, como por exemplo: a adoção de uma agenda em comum, o uso de lembretes colados na geladeira, a emissão de poucas informações por vez, assim como evitar fazer combinações quando houver distratores no ambiente (como, por exemplo, uma TV ligada). O cônjuge não afetado também sente muito alivio quando passa a ter uma explicação melhor para o problema do marido ou da esposa do que apenas "ele/ela não me ama" ou "ele/ela não me dá atenção".

Abordagens que dão ênfase à solução de problemas no trabalho são de grande valia. Estes pacientes apresentam grandes dificuldades de organizar o seu tempo e de dar prioridade a trabalhos em ordem hierárquica. Pacientes com TDAH apresentam muitas dificuldades para iniciar e terminar as suas tarefas, acumulando trabalhos inacabados ao longo do dia. É muito útil ajudar estes pacientes a fazerem uso de suas agendas - muitas vezes compradas no inicio do ano, porém nunca abertas. Também, orientar o paciente a procurar um espaço silencioso pode melhorar o seu nível de concentração e atenção. Muitos pacientes com TDAH apresentam melhor desempenho em trabalhos que demandem menos esforço mental. Por exemplo, aqueles pacientes com hiperatividade podem render mais em trabalhos que requeiram esforço físico e naquelas atividades ao ar livre.

\section{PROPOSTA DE UMA ESTRUTURA}

\section{PEDAGÓGICA PARA GRUPOS PSICOEDUCATIVOS EM TDAH}

A partir do Modelo de Estrutura e Planejamento Didático proposto por Antoni Zabala ${ }^{20}$, os autores do presente trabalho formulam uma proposta de estrutura pedagógica para grupos psicoeducacionais em TDAH. Pretende-se, em um futuro próximo, a utilização desta técnica no Ambulatório de adultos com TDAH do Hospital de Clínicas de Porto Alegre. Quando da sua implementação, os resultados serão objeto de publicação.

A proposta inicial é de que sejam utilizados os seguintes parâmetros para organizar as aulas/sessões:

1. Seqüência das atividades;

2. Papel dos professores/terapeutas e dos alunos/pacientes;

3. Organização social do grupo/aula;

4. Utilização dos espaços e do tempo das sessões;

5. Organização dos conteúdos;

6. Materiais didáticos;

7. Avaliação.

Seqüência das Atividades: As atividades didáticas (sessões) serão realizadas em quatro encontros com freqüência semanal. As sessões/aulas serão ministradas para grupos de dez pacientes. Na primeira sessão, serão discutidas as crenças dos pacientes sobre o TDAH. A segunda sessão/aula será utilizada para desfazer crenças irracionais sobre a doença e seu tratamento (equilíbrio entre as teorias dos pacientes e a realidade). A terceira e a quarta sessão/aula serão destinadas para discutir as dificuldades que os sintomas trazem para a vida dos pacientes e a maneira de enfrentá-los. No quarto encontro, será realizada a avaliação dos conteúdos expostos nas sessões/ aulas anteriores.

Papel dos professores/terapeutas e dos alunos/pacientes: Não haverá uma hierarquia rígida durante as sessões. Os terapeutas terão o papel de facilitar o desenvolvimento de uma cultura e um conhecimento sobre a doença. 0 papel dos pacientes/alunos também não será um papel passivo. Estes terão que construir o conhecimento sobre a doença. Os pacientes serão incentivados a ler sobre a doença em materiais fornecidos pela equipe ou em materiais disponíveis no mercado para o público leigo, a propor estratégias para lidar com suas limitações e sintomas e a ajudar os colegas a 
lidar com os efeitos colaterais através de uma rede de ajuda intra-grupo que será desenvolvida durante a duração do trabalho.

Organização Social do grupo/aula: O grupo de trabalho será composto de homens e mulheres encaminhados pelo ambulatório de Transtorno de Déficit de Atenção/hiperatividade do Hospital de Clínicas de Porto Alegre. O ideal seria formar grupos de pessoas que apresentassem escolaridade semelhante, no intuito de o grupo possuir homegeneidade. O nível sócioeconômico dos pacientes não será controlado, cabendo ao grupo identificar as diferentes necessidades dos integrantes. Isto poderá ser um fator facilitador para criar uma identidade dos participantes do grupo. De modo geral, os pacientes deste ambulatório pertencem às classes $A$ e $B$ pelos critérios do IBGE. A participação dos pacientes deverá ser estimulada pelos terapeutas, e estes deverão estar capacitados a desfazer os possíveis problemas de liderança negativa que venham a surgir. $O$ papel dos terapeutas será o de ministrar os conteúdos e o de dirigir o grupo em um processo de aquisição dos conhecimentos.

Utilização do tempo: As sessões/aulas terão a duração de uma hora. Não poderá ser excedido este tempo, já que é o período de atendimento concedido pelo Hospital de Clínicas de Porto Alegre à prática de tratamento em grupo. Os pacientes com TDAH apresentam grande dificuldade de ter uma noção realista de tempo e de organização, desta forma podem se beneficiar de que a duração das sessões/aulas seja limitada. Os terapeutas deverão advertir desde o início do tratamento sobre a duração das sessões/aulas e do número de sessões totais do tratamento proposto. Isto auxiliará no sentido de que os próprios pacientes possam administrar o seu tempo. As falhas, que porventura venham a ocorrer, devem ser utilizadas como exemplos práticos das dificuldades de organização.

Organização dos conteúdos: Como a proposta é a de fazer com que os pacientes criem, a partir de suas próprias experiências, os conteúdos a serem discutidos, não haverá uma seqüência formal de tópicos a serem abordados. Contudo, conhecimentos sobre sintomas, etiologia, curso, diagnóstico, genética, tratamentos, medicações utilizadas, efeitos colaterais comuns a estas drogas deverão ser apresentados pelos terapeutas ao grupo.

Material didático: Os pacientes receberão material impresso sobre TDAH e serão encorajados a lerem os materiais didáticos sobre TDAH disponíveis no mercado. Aqueles pacien- tes com acesso à internet serão também encorajados a trazer materiais disponíveis na rede.

Avaliação: No final das cinco sessões, haverá uma avaliação escrita sobre os conteúdos discutidos durante o curso do tratamento. Esta avaliação visa a individualizar as possíveis faIhas no aprendizado sobre os conteúdos referentes ao TDAH. Não é infreqüente que crenças não realistas determinem um mal desfecho no tratamento dos transtornos psiquiátricos. Por exemplo, a crença de que as medicações psiquiátricas são prejudiciais a longo prazo fazem com que muitos pacientes não tenham adesão ao tratamento.

\section{DISCUSSÃO}

O processo de ensino, segundo Zabala ${ }^{20}$, implica construção de um conhecimento previamente inexistente. A construção e a aquisição de um novo conhecimento implicam revisão e modificação de esquemas de conhecimento já adquiridos. Para que isto ocorra, não basta que o aluno se encontre frente a conteúdos para aprender. Faz-se necessário que este possa atualizar seus esquemas de conhecimento, compará-los com o que é novo, identificar semelhanças e diferenças e integrá-las em seus esquemas anteriormente incorporados. Quando todo este processo ocorre, pode-se dizer que está se produzindo uma aprendizagem efetiva. Os processos psicoterapêuticos não deixam de ser um processo pedagógico, no qual os pacientes aprendem novas alternativas para seus esquemas psicológicos anteriormente adquiridos. Geralmente, os esquemas psicológicos são aprendidos a partir das vivências ao longo da vida do paciente. Modificações nas suas cognições e comportamentos muitas vezes passam pelo aprendizado de novas cognições e novos comportamentos mais adaptativos. Para que isso ocorra, é necessário que o paciente construa com seu terapeuta as condições ideais desta mudança.

A proposta de uma abordagem psicoeducativa como um tipo de tratamento para adultos portadores de TDAH não possui a pretensão de obter mudanças transformadoras em apenas cinco encontros. O objetivo é o de iniciar, nestes pacientes, um processo terapêutico que a longo prazo permita uma adaptação mais adequada à vida familiar, social e laborativa. Já há evidências na literatura científica de que pacientes com depressão que se submetem a um tratamento psicossocial e psicoeducativo apresentam um índice de recaídas menores do que aqueles que apenas fazem uso de medica- 
ções ${ }^{21}$. Em crianças com TDAH, medidas educativas (apenas leitura de material didático sobre a doença) podem aumentar a adesão ao tratamento ${ }^{11}$

O objetivo do presente artigo foi o de propor uma nova abordagem psicoeducativa - que será implementada em um futuro próximo no Ambulatório de adultos com TDAH do Hospital de Clínicas de Porto Alegre. Esta abordagem leva em conta técnicas pedagógicas na criação de grupos psicoeducativos para pacientes portadores de TDAH, já que se sabe que esta população apresenta um grande número de problemas na área dos processos de ensino/aprendizado ${ }^{6}$. Isto faz com que esta população específica apresente dificuldades na aquisição de novos conteúdos, gerando inúmeros confilitos nas mais diversas áreas da vida destes pacientes. Desta forma, espera-se que este trabalho possa vir a colaborar em um futuro, sendo uma importante ferramenta neste campo de conhecimento - a interface entre a pedagogia e a psicoterapia, em especial no tratamento do TDAH na vida adulta.

\section{REFERÊNCIAS BIBLIOGRÁFICAS}

1. American Psychiatric Association. (1994). Diagnostic and Statistical Manual of Mental Disorders, Washington D. C., Fourth Edition, American Psychiatric Association.

2. Brown TE. (2000). Attention-deficit disorders and comorbidities in children, adolescents and adults. Washington, DC, American Psychiatric Press, Inc.

3. Biederman J, Mick E, Faraone SV. (2000). Age-dependent decline of symptoms of attention deficit hyperactivity disorder: impact of remission definition and symptom type. Am J Psychiatry. May;157(5):816-8.

4. Biederman J, Faraone SV, Lapey K. (1992). Comorbidity of diagnoseis. In: Attention-deficit hyperactivity disorder. New York, WB Saunders.

5. Barkley RA. (1998). Attention Déficit Hyperactivity Disorder: a handbook for diagnosis and treatment, $2^{\text {nd }}$ edition, New York, The Guilford Press.

6. Murphy KR, Barkley RA, Bush T. (2002). Young adults with attention deficit hyperactivity disorder: subtype differences in comorbidity, educational, and clinical history. $J$ Nerv Ment Dis. 190(3):147-57.

7. Mattos P, Belmonte-Abreu P, Grevet EH. (2003). O TDAH no adulto: dificuldades diagnósticas e de tratamento., ed ARTMED, Porto Alegre.

8. Pisterman S, McGrath P, Firestone P, Goodman JT, Webster I, Mallory R. (1989). Outcome of parent-mediated treatment of preschoolers with attention deficit disorde with hyperactivity. Journal of Consulting and Clinical Psychology, 57,628-635.

9. Horn WF, lalongo N, Greenberg G, Packard T, SmithWinberry C. (1990). Additive effects of behavioral parent training and self-control therapy with attention deficit hyperactivity disordered children. Journal of Clinical Child Psychology, 19:98-110.
10. Fehlings DL, Roberts W, Humphries T, Dawe G. (1991). Attention deficit hyperactivity disorder: Does cognitive behavioral therapy improve home behavior? Journal of Developmental and Behavioral Pediatrics. 12:223-228.

11. Long N, Rickert VI, Ashcraft EW. (1993) Bibliotherapy as an adjunct to stimulant medication in the treatment of attention-deficit hyperactivity disorder. Journal of Pediatric Health Care, 7:82-88.

12. Linden M, Habib T, Radojevic V. (1996). A controlled study of the effects of EEG biofeedback on cognition and behavior of children with attention deficit disorder and learning disabilities. Biofeedback and Self Regulation, $21: 35-49$.

13. Pfiffner LJ. \& McBurnett K. (1997). Social skills training with parent generalization: Treatment effects for children with attention deficit disorder. Journal of Consulting and Clinical Psychology, 65:749-757.

14. Schmidt MH, Mocks $P$, Lay B, Eisert HG, Fojkar R, FritzSigmund D, Marcus A, Musaeus B. (1997). Does oligoantigenic diet influence hyperactive/conduct-disordered children?: A controlled trial. European Child and Adolescent Psychiatry, 6:88-95.

15. Wilens TE, McDermott SP, Biederman J, Abrantes A, Hahesy A, Spencer TJ. (1999). Cognitive Therapy in: the Treatment of Adults With ADHD: A Systematic Chart Review of 26 Cases. Journal of Cognitive Psychotherapy, 13(3):126-29.

16. Hallowell EM e Ratey JJ. (1999). Tendência à distração: A identificação e a gerência do distúrbio do déficit de atenção da infância à vida adulta. Rio de Janeiro, Ed. Rocco.

17. Mattos P. (2001). No Mundo da Lua: Perguntas e respostas sobre Transtorno de Déficit de Atenção com Hiperatividade em crianças, adolescentes e adultos. São Paulo, Lemos Editorial.

18. Miller TW. (1989). Group sociotherapy: a psychoeducative model for schizophrenic patients and their families. Perspect Psychiatr Care, 25(1):5-9.

19. Anderson CM, Hugarty GF, Reiss DJ. (1980). Family treatment of schizophrenic patients: A psychoeducational approach. Schizophrenia Bulletin, 6(3):490-505.

20. Zabala A. (1998) A prática educativa: como ensinar. Porto Alegre, ed. ARTMED.

21. Scott J. (2003) Group psychoeducation reduces recurrence and hospital admission in people with bipolar disorder. Evid Based Ment Health. 6(4):115.

\section{RESUMO}

Os autores do presente artigo propõem, a partir do modelo pedagógico proposto por Antoni Zabala, uma abordagem psicoeducativa grupal para pacientes adultos portadores de Transtorno de Déficit de Atenção/Hiperatividade. Para tanto, adaptam esta estrutura pedagógica para fundamentar a abordagem de grupos psicoeducacionais. $O$ presente trabalho adiciona uma nova técnica para o tratamento do TDAH no adulto a partir da interface entre a pedagogia e a psicoterapia.

Descritores: Adultos, Grupos Psicoeducativos, pedagogia, Transtorno de Déficit de Atenção/Hiperatividade. 


\section{ABSTRACT}

TIn this paper the authors suggest a psychoeducative group approach to adult patients with Attention Deficit/Hyperactivity Disorder (ADHD) based on a pedagogic model proposed by Antoni Zabala. This paper intends to present this new technique for the treatment of adults with ADHD. The conceptualization is based on the interface between pedagogy and psychotherapy.

Key-words: Adults, psycho educative groups, pedagogy, attention Deficit Hyperactivity Disorder.

Title: A psychoeducacional approach in group therapy for adult patients with Attention Deficit/Hyperactivity Disorder

\section{RESUMEN}

Los autores del presente artículo proponen, a partir del modelo pedagógico propuesto por Antoni Zabala, un abordaje psico-educativo grupal para pacientes adultos portadores del Tratamiento del Déficit de Atención/Hiperactividad. Para tanto, adaptan esta estructura pedagógica para fundamentar el abordaje de grupos psico-educacionales. El presente trabajo adiciona una nueva técnica para el tratamiento del $T D A H$ en el adulto a partir de la interfase entre la pedagogía y la psicoterapia.

Palabras-clave: Adultos, Grupos psicoeducativos, Pedagogía, Transtorno de Déficit de Atención/Hiperactividad.

Título: Propuesta de un abordaje psico-educacional en grupos para pacientes adultos con trastorno de déficit de Atención/ hiperactividad

Endereço para correspondência:

Eugenio Grevet

Av. Taquara, 586/606

90460-210 - Porto Alegre - RS

E-mail: grevet.ez@terra.com.br

Copyright $\odot$ Revista de Psiquiatria do Rio Grande do Sul - SPRS 